\title{
Aplicação da Teoria de Valores Extremos e da Análise Fundamentalista em Estratégias Long-Short: uma Análise de Pair Tradings do Mercado Brasileiro
}

\section{Resumo}

Nas últimas décadas, tem crescido o número de fundos que procuram explorar ineficiências de mercado por meio de estratégias de arbitragem, nas quais se destaca a estratégia longshort. Grande parte das análises utilizadas para a obtenção dos pair tradings, entretanto, não levam em consideração os desvios extremos existentes no processo de interdependência entre os ativos envolvidos e os indicadores de qualidade operacional das firmas. A Teoria dos Valores Extremos e a Análise Fundamentalista foram utilizadas neste trabalho para que fossem modeladas as séries da razão entre preços de pares de ativos obtidos a partir da estrutura de indicadores contábeis propostos por Piotroski (2000). Tais abordagens permitiram que fossem consideradas empresas com sinalizações positivas de lucratividade, estrutura de capital e eficiência operacional, além de distribuições capazes de capturar os comovimentos extremos associados aos pair tradings selecionados. A partir de tal modelagem, foi criada uma nova abordagem quantitativa para a estratégia long-short, a qual denominamos estratégia GEV Long-Short. Os resultados obtidos sugerem que o melhor ajuste dos quantis extremos por intermédio da distribuição de valores extremos tem capacidade de fornecer sustentação probabilística mais refinada para que o retorno à média justifique $\mathrm{a}$ possibilidade de arbitragem long-short.

Palavras-Chave: Estratégias Long-Short; Teoria de Valores Extremos; Arbitragem; Pair Trading; Análise Fundamentalista.

\begin{abstract}
Danilo Soares Monte-Mor Mestre em Economia (UFES) e Doutorando em Ciências Contábeis e Administração (Fucape) e professor da Fucape Business School. Contato: Av. Fernando Ferrari, 1358, Boa Vista, Vitória (ES), CEP 29075-505. E-mail: danilo@fucape.br
\end{abstract}

Marco Aurélio dos Santos Sanfins Doutor em Estatística (UFRJ). Professor da Universidade Federal Fluminense (UFF). Contato: Rua Mário Santos Braga, S/N, Valonguinho, Niterói (RJ), CEP 24.020-140. E-mail: marcosanfins@automata.uff.br

\section{Silvania Neris Nossa}

Mestre em Ciências Contábeis (Fucape) e Doutoranda em Ciências Contábeis e Administração (Fucape). Contato: Av. Fernando Ferrari, 1358, Boa Vista, Vitória (ES), CEP 29075-505.

E-mail: silvania@fucape.br

\section{Aridelmo José Campanharo} Teixeira

Doutor em Controladoria e Contabilidade (USP). Professor da Fucape Business School. Contato: Av. Fernando Ferrari, 1358, Boa Vista, Vitória (ES), CEP 29075-505.

E-mail: aridelmo@fucape.br 


\section{Introdução}

Nas últimas décadas, tem crescido o número de fundos que procuram explorar ineficiências de mercado por meio de arbitragem. A exploração de tais ineficiências pode se dar por meio de estratégias como a estratégia long-short, na qual são assumidas tanto posições compradas (long) quanto vendidas (short) em ações ou índices de ações, seja no mercado à vista ou de derivativos. Por muitos anos, entretanto, grande parte dos pair tradings utilizados nessas estratégias foram construídos com base em análises que não consideram os desvios extremos existentes no processo de interdependência entre os ativos envolvidos e os indicadores de qualidade operacional das firmas. O objetivo deste trabalho é verificar se por meio da análise fundamentalista proposta por Piotroski (2000) e da Teoria de Valores Extremos é possível se estabelecer uma nova abordagem quantitativa para a estratégia long-short, de forma que sejam considerados, além de empresas com sinalizações positivas de lucratividade, estrutura de capital e eficiência operacional, distribuições capazes de capturar comovimentos extremos associados aos pair tradings selecionados.

Trabalhos que incluem Gatev, Goetzmann e Rowwenhorst (2006) se baseiam em abordagens na qual os comovimentos entre dois ativos é medido pela soma do quadrado das diferenças entre suas respectivas séries de preços normalizadas. Com abordagens semelhantes seguem Nath (2006), e Do e Hamza (2006). Percebe-se, entretanto, que a modelagem probabilística da série dos comovimentos dos preços dos pair tradings requer distribuições com caudas pesadas, uma vez que grande parte das oportunidades de arbitragem é gerada a partir desses movimentos extremos.

Outros trabalhos, como os de Alexander (1999), Alexander e Dimitriu (2005a), Dunis e Ho (2005) e Caldeira e Portugal (2010), utilizaram a técnica de cointegração, que procura explorar a dependência de longo prazo existente entre séries temporais, no contexto de estratégias index tracking. Herlemont (2000), Alexander e Dimitiu (2005b) e Lin, McRae e Gulati (2006) também aplicaram tal metodologia no contexto de estratégias que envolvem pair tradings. Percebe-se, entretanto, que, mesmo pares de ativos que não possuem interdependência de longo prazo, podem gerar em curto prazo janelas de arbitragem. Nesse sentido, faz-se necessário o desenvolvimento de técnicas quantitativas long-short mais apropriadas à análise dos comovimentos extremos dos pares de ativos, cointegrados ou não. É nesse contexto que a Teoria dos Valores Extremos desempenha um papel funamental dada a sua grande capacidade de modelagem de dados extremos.

Com o intuito de verificar a possibilidade da utilização da teoria de extremos na deteç̧ão de timings mais oportunos para arbitragem, foram selecionadas no mercado brasileiro empresas Winners com maior liquidez e valor de mercado. Segundo Piotroski (2000), ao considerarem-se portfólios com tais características, retornos anormais buy-and-hold de até $23 \%$ poderiam ser obtidos no mercado americano. Os resultados de Piotroski (2000) foram confirmados também no mercado brasileiro por Lopes e Galgi (2007), especialmente no segundo ano após a construção do portfólio. A partir de tais resultados, espera-se que a utilização da Análise Fundamentalista em consonância com a Teoria de Valores Extremos permita que sejam consideradas, além de empresas com sinalizações positivas de lucratividade, estrutura de capital e eficiência operacional, distribuições capazes de capturar os comovimentos extremos associados aos pair tradings selecionados.

Pares de ações de empresas importantes no cenário brasileiro foram utilizados para a aplicação da nova abordagem quantitativa a ser proposta, a qual denominamos estratégia GEV Long-Short. Os resultados obtidos foram comparados aos resultados que são obtidos quando os ajustes são feitos a partir da distribuição normal. A implementação da estratégia GEV Long-Short foi feita pela abordagem Block Máxima. Os parâmetros foram estimados por máxima verossimilhança. A estimação dos parâmetros e demais análises foram feitas com o uso do programa estatístico R-Project a partir do pacote fExtremes, específico para análise dos valores extremos.

Os resultados obtidos sugerem que a escolha de pairs trading, por meio da análise fundamentalista, e a modelagem das séries dos quocientes dos preços, por intermédio da distribuição de valores extremos, têm capacidade de forncecer um melhor ajuste dos quantis de probabilidades extremos, de forma a se detectar com uma maior precisão timings mais oportunos para as reversões das tendências de queda e de aumento da razão de precificação entre pares de ativos. 
Os resultados obtidos sugerem ainda que, embora possa existir um sincronismo aparente entre os preços das ações, a condição de cointegração não é necessariamente um requisito para que estratégias long-short possam ser construídas, como é o caso da estratégia GEV Long-Short proposta. Tal fato se sustenta, uma vez que a modelagem probabilística da razão de precificação, por meio da distribuição de valores extremos, não procura estabelecer relação de dependência entre os processos geradores de cada série de preços, mas verificar probabilisticamente, a partir da existência de cada processo gerador, momentos em que a razão de precificação se posiciona em quantis extremos e se mostra fora dos padrões de normalidade.

Este artigo contribui para a literatura de finanças ao propor a utilização da modelagem de dados extremos como ferramenta que pode fornecer informações para que seja feito um monitoramento ex-ante da difusão gradual das informações privadas e dos respectivos ajustes relativos dos preços de pares de ações. Tal monitoramento torna-se importante na medida em que possibilita que os players revejam suas expectativas (Análise Fundamentalista) e percebam com menor defasagem temporal o timing oportuno em que devem operar como traders (Teoria dos Valores Extremos). A modelagem de eventos extremos confere à abordagem proposta um avanço em termos de oportunidades de arbitragem com relação à metodologia que utiliza cointegração, uma vez que o fato de séries de preços não serem cointegradas não exclui a possibilidade da existência de janelas de arbitragem em curto prazo.

Este artigo segue estruturado da seguinte forma: nas seções 2 e 3 são feitas breves introduções acerca da estratégia Long-Short e da Teoria de Valores Extremos; na seção 4, é introduzida a estratégia GEV Long-Short e são apresentados os resultados comparativos entre os ajustes feitos pela distribuição de valores extremos e pela distribuição normal. A seção 5 se destina à conclusão e demais comentários.

\section{Hedge Funds e Estratégias Long-Short}

Dentre os fundos que utilizam a estratédia long-short destacam-se os Hedge Funds. Criado em 1949 por Alfred W. Jones, o primeiro Hedge Fund era um fundo cuja estratégia baseava-se na compra de ações subavaliadas e na venda a descoberto de ações sobreavaliadas com o intuito de proteger a carteira contra riscos de mercado, ou seja, reduzir o risco das carteiras por meio de posições vendidas em outras ações (Fothergill \& Coke, 2000). Assim, ao comprar ações que ele acreditava terem bom potencial de retornos e manter posição vendida em papéis nos quais estava pessimista, Jones acabou reduzindo a sua exposição ao risco do mercado de ações.

Ao trabalhar com tais posições, Jones reduzia seu risco, uma vez que, caso o mercado como um todo performasse de forma negativa, o fundo ganharia ao menos em parte de suas posições (no caso, as vendidas, ou short). Sua estratégia, entretanto, quando confirmadas as perspectivas traçadas, permitia que o fundo auferisse lucro nas duas operações, ou seja, com a alta das ações compradas e com a queda das vendidas a descoberto, uma vez que sua posição permitia adquiri-las por valor inferior. Essa estratégia fez com que, mesmo com a cobrança de uma taxa de remuneração em torno de $20 \%$ do retorno líquido, o fundo administrado por Jones apresentasse retorno maior que o de qualquer outro fundo, o que despertou grande interesse e atraiu, no final da década de 60 , tanto investidores quanto profissionais de mercado, que criaram centenas de novos fundos hedge (Fothergill \& Coke, 2000).

Com a crise da década de 70, entretanto, alguns Hedge Funds foram extintos e apenas na metade da década de 90 foram reintroduzidos nos fundos de interesse dos investidores (Anjivel, Boudreu, Perskin \& Urias, 2000). Mas se, teoricamente, as estratégias de investimentos adotadas por esse tipo de fundo reduziam os riscos, por que alguns investidores fazem associação dos Hedge Funds com investimentos de risco elevado e por que eles sofreram tanto com a crise da década de 70? A resposta reside no fato de que o objetivo da estratégia mencionada acima pode não ser o único adotado por um Hedge Fund.

Por exemplo, muitos Hedge Funds adotam estratégias que envolvem alavancagem, ou seja, o uso de derivativos para multiplicar o desempenho do fundo. Foi exatamente a adoção de estratégias mais agressivas e de posições extremamente alavancadas que levaram muitos Hedge Funds a fortes perdas no final da 
década de 60 e início da década de 70 nos EUA. Esse processo de acumulação de perdas durou até 1986, quando o desempenho do Tiger Fund colocou os Hedge Funds de volta como opção para grandes investidores (Branco \& Franco, 2004).

No Brasil, não existe regulamentação para os Hedge Funds, mas para fundos que, por vezes, atuam como Hedge Funds. De acordo com a nova classificação feita pelo Banco Central e baseada em risco (introduzida em março de 2005), tais carteiras estão entre os fundos genéricos. Fundos dessa categoria têm permissão de manterem posições alavancadas (Branco \& Franco, 2004). Nos EUA, os Hedge Funds são sociedades limitadas quanto ao número de cotistas, pouco reguladas e isentas dos controles da regulamentação Company Act de 1940 (restrições a alavancagem, venda a descoberto, concentração de risco, etc.), aplicada aos mutual funds (Liang, 2003). A falta de exigências quanto à transparência dos balanços e das operações dos Hedge Funds norte-americanos faz com que haja auditorias particulares. Essas auditorias, entretanto, segundo Liang (2003), são limitadas, uma vez que 40\% de uma amostra ampla não é auditada adequadamente. No Brasil, a rigidez na regulamentação das operações dos Hedge Funds é muito maior, uma vez que esses fundos recebem o mesmo tratamento legal que outros fundos, como os de renda fixa, por exemplo. Essa regulamentação, embora em alguns casos apresente restrições quanto às negociações, proporciona aos investidores maior segurança quanto à precificação das cotas dos respectivos fundos.

Atualmente, embora os Hedge Funds apresentem outras estratégias de investimento bastante diversificadas quanto ao grau de especialização e categorização (opportunistic, event driven, futures and currencies arbitrage, market timming, market neutral, global, equity hedge, etc.), a importância de Alfred W. Jones deve ser reconhecida, uma vez que, a partir de seu fundo hedge, estabeleceu-se o que hoje é conhecido como a estratégia long-short no mercado de ações.

Na próxima seção, serão apresentados os principais fundamentos da Teoria de Valores Extremos e da Análise Fundamentalista, as quais foram utilizadas na construção de uma nova abordagem quantitativa para a estratégia long-short.

\section{Análise Fundamentalista e a Teoria dos Valores Extremos (TVE)}

No universo quantitativo da detecção de janelas de arbitragem, não é conveniente considerar que todos os investidores estejam de acordo quanto à distribuição de probabilidade dos preços dos ativos. $\mathrm{O}$ que os investidores fazem é aproximar, em função das informações que têm disponíveis, a distribuição empírica dos dados para distribuições conhecidas e que melhor se adequem ao tipo de análise requerida. Dessa forma, em curto prazo, os preços podem não refletir todas as informações existentes, mas o conjunto de interpretações que cada investidor faz das informações publicamente disponíveis (ineficiências de curto prazo). Por consequência, são criadas janelas de arbitragem, dadas as diferentes expectativas que cada investidor faz acerca da estratégia e da carteira ótima para alocação de seus recursos.

A assimetria informacional existente entre o gestor e os acionistas que não participam diretamente das tomadas de decisões, por exemplo, é um dos fatores que pode justificar as possibilidades de arbitragem. $\mathrm{Na}$ literatura de finanças, tem se tornado mais frequente a utilização da contabilidade para a diminuição de tal assimetria informacional. Alguns estudos buscaram relacionar os retornos das ações com indicadores financeiros construídos a partir de informações contábeis (análise fundamentalista), de forma que fossem verificada a relação existente entre os preços e informações contidas nos balanços das empresas: Ball e Brown (1968), Fama e French (1992), Baruch e Thiagarajan (1993), Fama e French (1995), Fama e French (1996), Abarbanell e Bushee (1997), Abarbanell e Bushee (1998), Ali e Hwang (2000), Bird, Gerlach e Hall (2001), Piotroski (2000), e Lopes e Galdi (2007). Tais estudos sugerem que a transparência e qualidade tanto dos processos de gestão como das informações contidas nos balanços são fatores associados ao desempenho das empresas no mercado de ações. Com base em tais características, Piotroski (2000) construiu portfólios no mercado americano com base em empresas que foram classificadas como winners e losers. 
Na próxima seção, será verificado que é possível, por meio da análise fundamentalista proposta por Piotroski (2000) e da Teoria de Valores Extremos, estabelecer uma abordagem que permita que os players de mercado revejam suas expectativas (Análise Fundamentalista) e percebam com menor defasagem temporal o timing oportuno em que devem operar como traders.

\subsection{Modelagem Univariada dos Máximos}

A TVE é um ramo da probabilidade que estuda o comportamento estocástico de extremos associados a um conjunto de variáveis aleatórias com distribuição comum F. As características da distribuição desses extremos (distribuição do máximo ou mínimo) são determinadas pelas caudas da distribuição F.

Alguns autores se referem ao artigo de Bortkiewicz (1922) como o marco inicial do desenvolvimento da Teoria dos Valores Extremos (TVE). Nesse artigo, Bortkiewicz aborda a distribuição do tamanho do intervalo entre o máximo e o mínimo, mas em uma amostra com distribuição normal. Em termos mais gerais, os fundamentos básicos da TVE foram inicialmente expostos por Fisher e Tippett (1928), que introduziram os três tipos possíveis de distribuição assintótica dos valores extremos, hoje conhecidas como distribuições de Gumbel, Fréchet e Weibull. Entretanto, um dos primeiros a estudar e a formalizar a aplicação estatística dessa teoria foi Gumbel (1954), cuja metodologia será apresentada a seguir.

Ao se analisar uma amostra como um todo, encontra-se apenas um máximo (ou mínimo) absoluto associado a um número finito de observações. No intuito de tornar a disposição das observações passível de análise a partir da TVE, uma amostra pode ser dividida em subperíodos disjuntos e de mesmo tamanho, de forma que sejam extraídos os máximos de cada subperíodo.

Considere, para tanto, variáveis aleatórias $\mathrm{X}_{1}, \mathrm{X}_{2}, \ldots$ i.i.d. com função de distribuição $\mathrm{F}$ e um conjunto $\mathrm{X}=\left\{\mathrm{X}_{1}, \mathrm{X}_{2}, \ldots, \mathrm{X}_{\mathrm{n}}\right\}$ formado por $n$ dessas variáveis. A partir desse conjunto podemos obter uma nova sucessão ao rearranjarmos seus termos em ordem crescente de magnitude, a saber

$$
\left(\mathrm{X}_{(\mathrm{n})}\right)=\left\{\mathrm{X}_{(1)}, \mathrm{X}_{(2)}, \ldots, \mathrm{X}_{(\mathrm{n})}\right\}
$$

em que $X_{(1)}=\min X_{\text {e }} X_{(n)}=\max X$

Definição 3.1 - A função $F \mathrm{x}_{(n)}(x)=P\left(X_{(n)} \leq x\right)=[P(X \leq x)]^{n}=F^{n}(x), x \in \mathbb{R}, n \in \mathbb{N}$ é dita função de distribuição de máximo.

Note que na definição acima $P\left(\mathrm{X}_{(n)} \leq x\right)=[P(\mathrm{X} \leq x)]^{n}$, uma vez que, dada a independência das variáveis $P\left(\cap_{i=1}^{n}\left[X_{i} \leq x\right]=\mathbb{M}_{i=1}^{n}\left[P\left(\mathrm{X}_{i} \leq x\right)\right]\right.$.

Observação 3.1 - Embora na maior parte das vezes tratemos à TVE a partir de uma abordagem das observações máximas, os mesmos resultados podem ser utilizados na abordagem dos mínimos, dada a conversão imediata

$$
\operatorname{Min}\left(X_{1}, X_{2}, \ldots X_{n}\right)=-\operatorname{Max}\left(-X_{1},-X_{2}, \ldots-X_{n}\right)
$$

A partir da distribuição do máximo e interessados na análise da cauda da distribuição $\mathrm{F}$, observe que o máximo converge em probabilidade para o limite superior $\mathrm{x}_{\mathrm{F}}$ do suporte da distribuição $\mathrm{F}$, ou seja

$$
X_{(n) \stackrel{p}{\rightarrow}} x_{F}
$$

quando $\mathrm{n} \rightarrow \infty$ e para $x_{F} \leq \infty$, sendo $x_{F}=\sup \{x \in \mathbb{R} / F(\mathrm{x})<1\}$, uma vez que para $n$ suficientemente grande: 
- $\quad$ se $\mathrm{x}<x_{F}$, então $P\left(\mathrm{X}_{(n)} \leq x\right)=F^{n}(x) \rightarrow 0$

- $\quad$ se $\mathrm{x} \geqslant x_{F}, \operatorname{com} x_{F}<\infty$, então $P\left(\mathrm{X}_{(n)} \leq x\right)=F^{n}(x) \rightarrow 1$

Entretanto, para valores de $n$ pequenos, é necessário que se conheça previamente a distribuição $\mathrm{F}$ (que em geral é desconhecida) para que se estabeleça a distribuição do máximo e, para valores de $n$ suficientemente grandes, a função de distribuição do máximo torna-se degenerada, ou seja, $P\left(\mathrm{X}_{(n)} \leq x\right) \rightarrow 0$.

Fisher e Tipet (1928), contudo, estabeleceram um resultado no qual a distribuição dos máximos padronizados por sequências de constantes $\left(a_{n}\right)$ e $\left(b_{n}\right)$ converge para certas distribuições limite, denominadas distribuições extremas ou distribuições Extreme Value (EV). Esse resultado é hoje conhecido como teorema de Fisher-Tippett.

Teorema 3.1 (Teorema de Fisher Tippett) - Seja $\left(x_{n}\right)_{n \in \mathbb{N}}$ uma sucessão de variáveis aleatórias i.i.d com função de distribuição F. Se existir uma sequência $\left(a_{n}\right)$ de termos positivos, uma sequência real $\left(b_{n}\right)$ e uma função de distribuição $\mathrm{H}$ não degenerada tais que

$$
P\left[\mathrm{X}_{(n)} \leq a_{n} x+b_{n}\right]=F^{n}\left(a_{n} x+b_{n}\right) \stackrel{d}{\rightarrow} H(x)
$$

então as únicas formas possíveis de H são as distribuições Gumbel, Fréchet ou Weibull, também denominadas distribuições do tipo I, II e III, respectivamente.

Tais distribuições limite estão associadas a um parâmetro de forma $\lambda$. A seguir estão apresentadas as distribuições limite para máximos, bem como os respectivos gráficos das distribuições extremas com parâmetro de forma iguais a 0,1 e -1 , respectivamente:

- Gumbel $\lambda=0, x \in \mathbb{R}): \mathrm{F}_{\lambda}(x)=\exp [-\exp (-x)]$

- Fréchet $\lambda>0, x \in \mathbb{R}): \mathrm{F}_{\lambda}(x)=\left\{\begin{array}{cc}\exp (-x)^{-\lambda}, & \text { se } x>0 \\ 0, & \text { se } x \leq 0\end{array}\right.$

- Weibull $(\lambda<0, x \in \mathbb{R}): \mathrm{F}_{\lambda}(x)=\left\{\begin{array}{cc}\exp \left[-(-x)^{-\lambda}\right], & \text { se } x \leq 0 \\ 1 & \text { se } x>0\end{array}\right.$

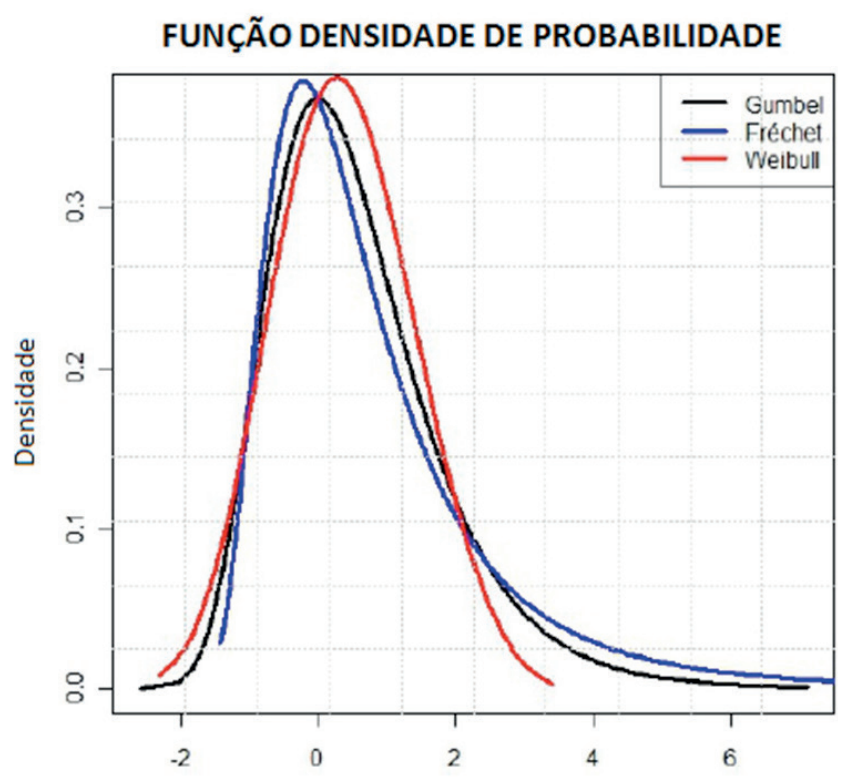

Figura 1. Gráfico das distriuições extremas com parâmetros de forma iguais a 0, 1 e -1, respectivamente 
Note que o Teorema de Fisher-Tippett especifica as distribuições extremas limite para as quais há possibilidade de convergência de distribuição do máximo padronizado $\frac{X_{(n)}-b_{n}}{a_{n}}$, embora não estabeleça condições que F deve satisfazer para que ocorra tal convergência (Embrechts, Klippelberg Mikosch, 1997).

Observe ainda que as distribuições EV podem ser estimadas sem que se faça necessária a utilização da distribuição F. Uma vez estimada a distribuição limite, entretanto, inferências acerca da distribuição empírica F podem ser feitas, uma vez que de $H(\mathrm{x})=F^{n}(\mathrm{x})$ obtém-se:

$$
F(\mathrm{x})=[H(x)]^{1 / n}
$$

Em trabalhos anteriores aos publicados por Von Mises (1936) e Jenkison (1955), entretanto, a estimação do parâmetro $\lambda$ que especifica a distribuição limite era feita, supondo-se a convergência da distribuição teórica F para cada uma das distribuições limite, com posterior aplicação de testes para verificação da distribuição extrema com melhor qualidade de ajuste. O avanço teórico introduzido por esses autores foi bastante significativo, uma vez que sob a $\xi$-parametrização, ou representação de Jenkison-van Mises 1936 e Jenkison (1955), as distribuições de Gumbel, Frichét e Weibull podem ser generalizadas em uma forma denominada Distribuição Generalizada de Valor Extremo (Generalized Extreme Value - GEV), que representa uma família de funções de distribuição de um único parâmetro $\xi$. Ou seja, a parametrização das funções estremas permitiu que fossem estimados os parâmetros da verdadeira distribuição limite. Segue a Distribuição Generalizada de Valor Extremo:

$$
G_{\xi}(T(x))=G_{\xi, \mu, \sigma}(x)=\left\{\begin{array}{l}
\exp \left(-\left(1+\xi \frac{x-\mu}{\sigma}\right)^{\frac{-1}{\xi}}, \xi \neq 0, \quad 1+\xi \frac{x-\mu}{\sigma}>0\right) \\
\exp \left[-\exp \left(-\frac{x-\mu}{\sigma}\right)\right], \xi=0
\end{array}\right.
$$

Para o caso limite $(\xi \rightarrow 0)$, temos que $\mathrm{G}_{\xi}$ corresponde à distribuição Gumbel. Ainda, se $\xi<0, \mathrm{H}_{\xi}$ corresponde à distribuição Weibull e se $\xi>0, \mathrm{H}_{\xi}$ corresponde à distribuição Fréchet.

A função densidade de probabilidade (fdp) da distribuição generalizada $G_{\xi \mu \sigma}$ pode ser obtida por diferenciação e aplicação da regra da cadeia segue abaixo:

$$
G_{\xi, \mu, \sigma}(x)=\left\{\begin{array}{l}
\left(1+\xi \frac{x-\mu}{\sigma}\right)^{\left(\frac{-1}{\xi}-1\right)} \exp \left[-\left(1+\xi \frac{x-\mu}{\sigma}\right)^{\frac{-1}{\xi}}\right], \xi \neq 0, \mu \in R, \sigma>0 \\
\exp \left[-\exp \left(-\frac{x-\mu}{\sigma}\right)\right] \exp \left(-\frac{x-\mu}{\sigma}\right) \frac{1}{\sigma}, \xi=0, \quad x \in \mathbb{R}, \quad \mu \in \mathbb{R}, \quad \sigma>0
\end{array}\right.
$$

Na Figura 2 estão apresentados os gráficos das fdp associadas a cada uma das três distribuições. 


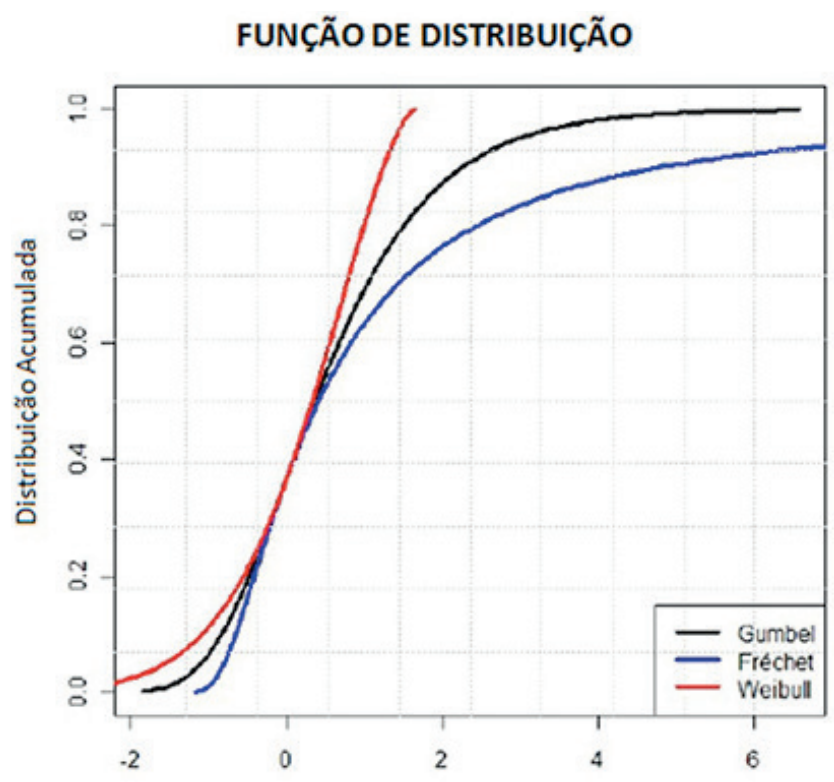

Figura 2. Gráfico fdp das distribuições extremas com parâmetro de forma iguais a 0,1 e -1 , respectivamente

\section{Nova Proposta Quantitativa Long-Short}

Essa seção se destina à apresentação da nova abordagem quantitativa long-short, construída a partir de pesquisa empírica em que foram utilizados dados de empresas listadas na BM\&FBovespa referente ao período entre 2005-2008. Além dos preços, foram utilizadas informações contábeis obtidas na base de dados Economática no intuito de selecionar as empresas Winners, conforme metodologia proposta por Piotroski (2000). Uma vez que o interesse é verificar a possibilidade da utilização da teoria de extremos na detecção do timming mais oportuno para arbitragem, foram selecionadas apenas as empresas Winners com maior liquidez e valor de mercado. Segundo Piotroski (2000), ao considerar-se tais portfólios, retornos anormais de até $23 \%$ poderiam ser obtidos em estratégias buy-and-hold. Os resultados de Piotroski (2000) foram confirmados também no mercado brasileiro por Lopes e Galdi (2007), especialmente no segundo ano após a construção do portfólio. A Tabela 1 apresenta os 9 indicadores utilizados na construção do score proposto por Piotroski (2000).

Das empresas pertencentes ao quintil superior (winners), foram selecionadas aquelas empresas de maior liquidez e valor de mercado (Lopes \& Galdi, 2007). A partir da modelagem feita pela Teoria dos Valores Extremos da razão dos preços das empresas selecionadas, as ações winners selecionadas foram monitoradas de forma que fosse verificado o momento em que a razão de preços atingisse os quantis-extremos fornecidos pela modelagem dos extremos. As empresas selecionadas foram monitoradas durante os dois anos após a formação do portfólio winner, conforme proposição de Lopes e Galdi (2007).

\subsection{Estratégia GEV Long-Short}

A estratégia long-short mais simples adotada pelos players, também chamada de estratégia number one, é um tipo de estratégia pair trading e está baseada no aluguel de uma determinada ação que está sobreavaliada em relação ao preço de outra ação específica (Nicholas, 2000). O objetivo dessa estratégia é obter um retorno adicional e que não esteja relacionado à apreciação ou depreciação desses ativos, mas ao desempenho relativo entre as posições compradas (long) e vendidas (short). 
Nessa operação, as ações alugadas são imediatamente vendidas e o montante obtido é totalmente investido na compra da outra ação que compõe o pair trading. Ao final do período de maturação do aluguel, espera-se que o pair trading esteja com razão de precificação $(\mathrm{k})$ em relação à posição inicial menor o suficiente para cobrir os custos gerais da transação e gerar os retornos adicionais desejados, ou seja, espera-se que a venda das ações compradas seja superior ao valor necessário para compra das ações alugadas e que devem ser devolvidas acrescidas da taxa de negociação, que em geral é de $1 \%$ (Nicholas, 2000). De modo análogo, caso k seja obtido por meio da razão dos preços dos ativos subavaliado e sobreavaliado, respectivamente, então se espera que a razão k esteja maior o suficiente para que se possa alcançar os resultados desejados.

Observe que, mesmo que o mercado opere em queda, é possível que se obtenha rentabilidade positiva. Basta que as posições sejam feitas de forma que o ativo short se desvalorize mais que o ativo long. Esse é, entre outros, um dos importantes benefícios que as estratégias long-short podem proporcionar, uma vez que, além de oportunizarem rentabilidades positivas, protejem a carteira contra riscos sistêmicos de declínio do mercado.

A grande questão que envolve a nova implementação quantitativa long-short proposta baseia-se na modelagem (por meio da TVE) da série obtida a partir do quociente dos preços do pair trading a ser arbitrado, de forma que seja detectado, por exemplo, com 95\% de confiança, o momento em que essa razão ocupe quantis extremos da GEV ajustada, ou seja, para que seja determinado a priori oportunidades de arbitragem entre pares de ativos.

Para tanto, neste estudo, foi utilizada a estratégia proposta por Piotroski (2000) para a seleção de empresas winners, utilizando-se informações contábeis de 2005 e 2006. Do portfólio Winner foram selecionadas as empresas com maior liquidez para que fossem contruídos os pair tradings, os quais foram monitorados durante os dois anos após a formação do portfólio winner (2007 e 2008), conforme proposição de Lopes e Galdi (2007).

Tabela 1

Indicadores e variáveis contábeis utilizados na construção do F-Score

\begin{tabular}{|c|c|c|}
\hline Indicador & Variável & Score \\
\hline \multirow{2}{*}{$\mathrm{ROA}_{i t}$} & \multirow{2}{*}{$\mathrm{LL}_{i t} / \mathrm{AT}_{i t-1}$} & $\uparrow \mathrm{ROA}>0(1)$ \\
\hline & & $\downarrow R O A<0(0)$ \\
\hline \multirow{2}{*}{$\mathrm{CF}_{i t}$} & \multirow{2}{*}{$\left(\right.$ CXECX $_{i t}-$ CXECX $\left._{i t-1}\right) / A T_{i t-1}$} & $\uparrow \mathrm{CF}>0(1)$ \\
\hline & & $\downarrow C F<0(0)$ \\
\hline \multirow{2}{*}{$\triangle \mathrm{ROA}_{i t}$} & \multirow{2}{*}{$\mathrm{ROA}_{i t}-\mathrm{ROA}_{i t-1}$} & $\uparrow \Delta \mathrm{ROA}>0(1)$ \\
\hline & & $\downarrow \Delta R O A<0(0)$ \\
\hline \multirow{2}{*}{$\mathrm{ACCRUAL}_{i t}$} & \multirow{2}{*}[\mathrm{LL}_{it}-(\mathrm{CXECX}_{it}-\mathrm{CXECX}_{it-1})]{$/ \mathrm{AT}_{i t-1}$} & $\uparrow \mathrm{CF}>\mathrm{ROA}(1)$ \\
\hline & & $\downarrow C F<R O A(0)$ \\
\hline \multirow{2}{*}{$\Delta \mathrm{LIQUIDEZ}_{i t}$} & \multirow{2}{*}{$\left(A C_{i t} / P C_{i t}\right)-\left(A C_{i t-1} / P C_{i t-1}\right)$} & $\uparrow \Delta \mathrm{LI} \mathrm{Q}>0(1)$ \\
\hline & & $\downarrow \Delta \mathrm{LIQ}<0(0)$ \\
\hline \multirow{2}{*}{$\Delta \mathrm{ENDIVID}_{i t}$} & \multirow{2}{*}[(\mathrm{PC}_{it}+\mathrm{PELP}\mathrm{P}_{it})/\mathrm{AT}_{it}]{$-\left[\left(\mathrm{PC}_{i t-1}+\mathrm{PELP} \mathrm{P}_{i t-1}\right) / \mathrm{AT}_{i t-1}\right]$} & $\uparrow \Delta$ ENDIVID $>0(1)$ \\
\hline & & $\downarrow \Delta$ ENDIVID $<0(0)$ \\
\hline \multirow{2}{*}{ OFERTA DE AÇÕES } & \multirow{2}{*}{ Emissão de ações no ano anterior à emissão do portfólio } & $\uparrow$ OFER $=0(1)$ \\
\hline & & $\downarrow$ OFER $>0(0)$ \\
\hline \multirow{2}{*}{$\triangle$ MARGEM $_{i t}$} & \multirow{2}{*}{$\left(\mathrm{LB}_{i t} / \mathrm{REC}_{i t}\right)-\left(\mathrm{LB}_{i t-1} / \mathrm{REC}_{i t-1}\right)$} & $\uparrow \Delta M A R G>0(1)$ \\
\hline & & $\downarrow \Delta M A R G<0(0)$ \\
\hline \multirow{2}{*}{$\Delta \mathrm{GIRO}_{i t}$} & \multirow{2}{*}{$\left(\mathrm{REC}_{i t} / \mathrm{AT}_{i t}\right)-\left(\mathrm{REC}_{i t-1} / \mathrm{AT}_{i t-1}\right)$} & $\uparrow \Delta \mathrm{GIRO}>0(1)$ \\
\hline & & $\downarrow \Delta \mathrm{GIRO}<0(0)$ \\
\hline
\end{tabular}

Obs.: O F-Score (Piotroski, 2000) é um indicador construído a partir da soma de Scores relacionados à indicadores contábeis referentes à lucratividade, estrutura de capital e eficiência operacional. De acordo com essa classificação, cada empresa i pode ter score mínimo de zero e máximo de nove em cada ano fiscal. As definições das variáveis que compõem os indicadores que fazem parte do F-Score seguem apresentados a seguir: 
- $\quad$ CXECX = Caixa e equivalente a caixa da empresa i no ano t;

- $A C=$ Ativo circulante da empresa i no ano t;

- $P C=$ Passivo circulante da empresa i no ano t;

- LB = Lucro bruto da empresa i no ano t;

- $\quad$ PELP = Passivo exigível em longo prazo da empresa i no ano t;

- $\mathrm{REC}=$ Receita de vendas da empresa i no ano t;

- $\mathrm{LL}=$ Lucro líquido da empresa i no ano t;

Fonte: Nossa, Teixeira e Lopes (2010, p. 8), adaptado de Piotroski (2000) e Lopes e Galdi (2007)

Observe que, para que possa ser feita a análise das séries de preços a partir da teoria de extremos, faz-se necessário que seja escolhido um período que contemple a existência de ambos os ativos, ou seja, de forma que tenhamos informações sobre os preços dos ativos para todas as datas do período escolhido. Na nossa análise, o período escolhido para a obtenção dos quantis extremos não incorpora a crise financeira de 2008, uma vez que essa crise gerou impactos singulares no comportamento de muitos ativos. Observe ainda que o número de dados coletados deve ser suficientemente grande para que possamos dividir a série do quociente dos preços do pair trading a ser arbitrado em blocos (abordagem Block Máxima).

Para que possa ser verificado o melhor ajuste da GEV e garantir a estabilidade da distribuição ajustada, foi feita uma análise comparativa por meio de modelagens com tamanhos de bloco iguais a 5, 10, 15 e 20 observações, de modo que sejam obtidas quatro séries com os valores das razões máximas e mínimas para períodos de 1, 2, 3 e 4 semanas, respectivamente.

A estatística de Kolmogorov-Smirnov foi utilizada para testar se a suposição de que a distribuição dos máximos e mínimos dos dados analisados convergem para a distribuição GEV. Uma análise dos resíduos por meio do QQ-Plot foi feita para que fosse verificada ainda a qualidade do ajuste da GEV para os diferentes tamanhos de blocos considerados.

Ajustada a GEV para as séries dos máximos e mínimos e verificado para qual tamanho de bloco se tem maior estabilidade, foram calculados os quantis $95 \%$ e 5\% para as séries dos máximos e mínimos referentes a esse tamanho de bloco, respectivamente. Esse ajuste, por meio da distribuição de valores extremos leva, à definição:

Definição 4.1 (Quantil GEV Long-Short) - Seja $\left\{X_{1}, X_{2}, \ldots, X_{n}\right\}$ um conjunto de variáveis aleatórias que representam a razão de precificação entre ativos. Denomina-se Quantil GEV Long-Short o quantil 95\% (ou 5\%) obtido por meio da distribuição extrema para o qual a distribuição dos máximos (ou mínimos) padronizados converge.

Os quantis GEV Long-Short, juntamente com os quantis fornecidos pela normal, foram superpostos à série das razões para um período subsequente ao período analisado. Como inferências acerca da verdadeira distribuição $\mathrm{F}$ da série podem ser feitas a partir da GEV ajustada, foram superpostos também nesse gráfico os verdadeiros quantis da série, ambos obtidos a partir da GEV estimada. Esses quantis foram utilizados para que fossem verificados os momentos nos quais a razão de precificação ocupou quantis extremos. A busca pelo timing de arbitragem mais oportuno leva à definição:

Definição (Saturação relativa do desequilíbrio) - Dois ativos encontram-se em posição de saturação relativa de desequilíbrio quando a razão de precificação desses ativos encontra-se fora do intervalo definido pelos quantis GEV Long-Short.

A partir da detecção de tais janelas (pontos de saturação relativa do desequilíbrio), foram estruturadas subestratégias de arbitragem que iniciem em posições compradas e vendidas e que liquidem ou aumentem as posições, a depender do comportamento dos retornos oferecidos pelo par de ativos arbitrado. Um nível de rentabilidade para a operação de $3 \%$ foi fixado como ponto de stop para a operação. Vale ressaltar que o nível de rentabilidade para o desarme da operação não necessariamente deve ser de $3 \%$, mas pode variar de acordo com o risco que o investidor está disposto a correr e com o percentual de rentabilidade desejado. 
A partir das informações obtidas e da elaboração das subestratégias, foram calculadas as rentabilidades fornecidas pela abordagem GEV e pela abordagem que utiliza distribuição Normal. A comparação de tais estratégias é essencial para que se confirme a suposição de que o melhor ajuste dos quantis extremos por intermédio da GEV tem capacidade de fornecer sustentação probabilística mais refinada para que o retorno à média justifique a possibilidade de arbitragem long-short.

Os pares de ativos winners PETR4 x VALE5 e VIVO4 x TCSL4 foram utilizados para a aplicação da nova abordagem quantitativa proposta. Observe que o primeiro caso é um exemplo de arbitragem entre empresas de setores diferentes e que conferem grande representatividade no total de negociações do mercado brasileiro. O segundo par se refere à arbitragem entre ativos winners de um mesmo setor, que no período apresentava em importante estágio de crescimento no Brasil.

Embora nos dois casos exista um sincronismo entre os preços do par de ativos considerados, tal sincronismo não é necessariamente um requisito para que estratégias long-short possam ser construídas, como é o caso da GEV Long-Short Strategy proposta. Tal fato se sustenta, uma vez que a modelagem probabilística da razão de precificação por meio da GEV não procura estabelecer relação de dependência entre os processos geradores de cada série de preços dos ativos, mas verificar probabilisticamente, a partir da existência de cada processo gerador, momentos nos quais a razão de precificação ocupa quantis extremos e se mostra fora dos padrões usuais. Essa interpretação confere à metodologia de análise um avanço em termos de oportunidades de arbitragem com relação à metodologia que utiliza, por exemplo, a cointegração, uma vez que o fato de séries de preços de ativos não serem cointegradas não exclui a possibilidade da existência de janelas de arbitragem no curto prazo. Pelo teste de Engle-Granger verificou-se que o par de ativos PETR4 x VALE5 não é cointegrado e que o par VIVO4 x TCSL4 é cointegrado.

\subsection{PETR4/VALE5 e VIVO4/TCSL4}

A seguir apresenta-se uma modelagem comparativa da razão entre os preços da PETR4 e VALE5 a partir do ajuste das distribuições GEV e Normal. Os dados coletados formam uma amostra de 651 observações dos valores diários dos preços desses ativos para o período de 1\%/1/2005 a 2/7/2007.

A partir do método de máxima verossimilhança, foram obtidas as estimativas para os parâmetros $\xi, \mu$ e $\sigma$ da GEV na modelagem dos máximos e mínimos, respectivamente, para blocos de tamanho 5, 10, 15 e 20 dias.

As Figuras 3 e 4 se referem ao QQ-plot dos resíduos para as modelagens referentes ao máximo e mínimo para os diferentes tamanhos de blocos. Note que na modelagem do mínimo os blocos de tamanho 20 garantiram maior estabilidade para a GEV, uma vez que o plot dos resíduos mostrou melhor ajuste. Já na modelagem dos máximos, os blocos de tamanho 15 garantiram maior estabilidade. A seguir estão apresentadas as estimativas dos parâmetros $\xi$, $\mu$ e $\sigma$ para a modelagem dos máximos e mínimos com blocos de tamanho 15 e 20 , respectivamente.

$$
\begin{array}{lll}
\xi=-0,3945844 & \mu=1,6959661 & \sigma=0,2533173 \\
\xi=-0,5216825 & \mu=1,5910817 & \sigma=0,2604460
\end{array}
$$

A estatística de Kolmogorov-Smirnov foi utilizada para se testar a suposição de que a distribuição do máximo e mínimo dos dados analisados converge para a distribuição GEV ajustada. Os p-valores obtidos na modelagem dos máximos e mínimos foram de 0,9429 e 0,9373, respectivamente, suficientes para garantirem as convergências. 


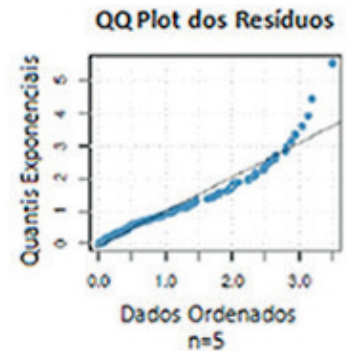

QQPlot dos Residuos

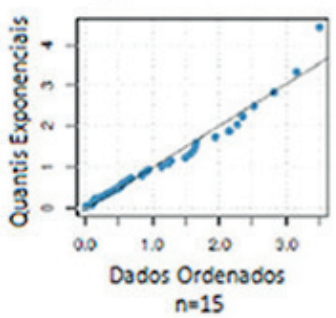

Figura 3. QQ-PLot dos resíduos para a modelagem dos máximos

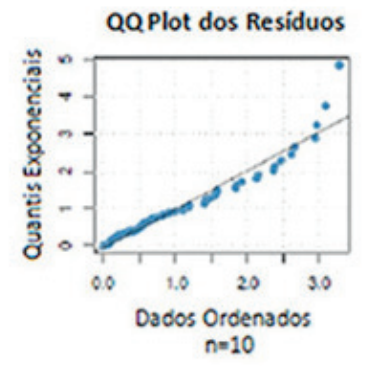

QQ Plot dos Residuos

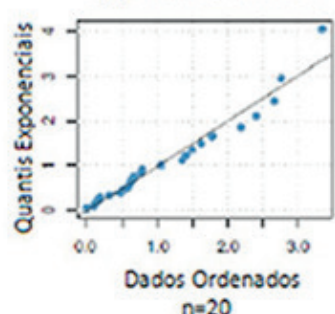

$n=20$
QQ Plot dos Residuos

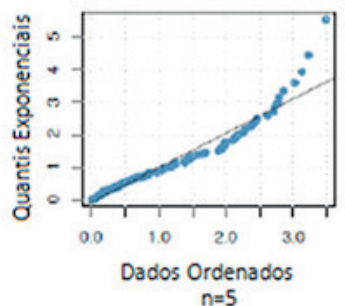

QQ Plot dos Residuos

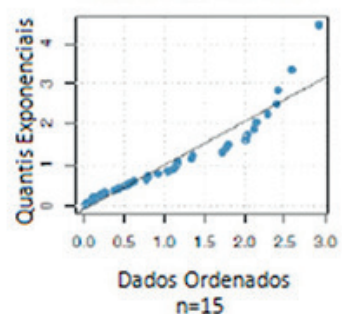

Figura 4. QQ-PLot dos resíduos para a modelagem dos minimos

A partir dos parâmetros obtidos, a GEV ajustada para mínimos e máximos fornece 1,183819 e 2,139097 como quantis 5\% e 95\% para a razão $\mathrm{k}=$ PETR4.SA/VALE5.SA, respectivamente. Ao utilizar-se uma distribuição Normal, obtêm-se para o mesmo nível de significância os valores 1,299182 e 2,109515, respectivamente. A Figura 5 compara os quantis de probabilidade obtidos com a performance da razão $\mathrm{k}$ para os 131 dias subsequentes ao período analisado. Note que, caso os players tivessem conhecimento acerca dos quantis estimados pela Normal e GEV, a partir do monitoramento diário da evolução da razão entre os ativos a seguinte estratégia de arbitragem poderia ser construída:
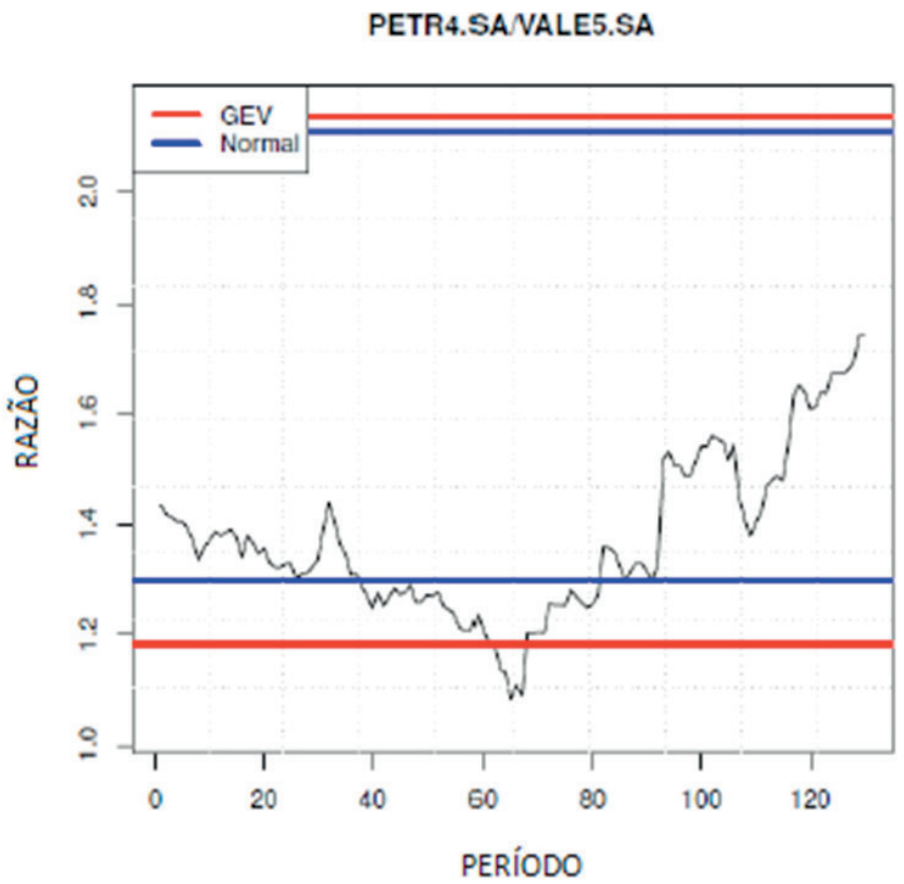

Figura 5. Comparação dos quantis de probabilidade obtidos com a performance da razão $\mathrm{k}=$ PETR4.SA/VALE5.SA para os 131 dias subsequentes a 02/07/2007 
1. Ao acompanhar-se a evolução da razão $\mathrm{k}=$ PETR4.SA/VALE5.SA, verifica-se que no dia 8/8/2007 (período 26), poder-se-ia montar posições comprada em PETR4 e vendida em VALE5, uma vez que nesse dia a razão $k$ atinge o quantil $5 \%$ estimado através da distribuição Normal;

2. Ao fixar uma rentabilidade acumulada de $3 \%$ como ponto de stop da operação, verifica-se que o desarme de tais posições aconteceria no dia 15/8/2007 (período 32), cinco dias úteis depois do start da operação (ver Figura 6);

3. Em razão de a modelagem pela normal sobre-estimar o quantil inferior, ao invés de simplemente desarmar-se a posição, os players poderiam inverter as posições, ou seja, assumir posição comprada em VALE5 e vendida em PETR4, uma vez que nesse período a razão k ainda não atingiu o suporte inferior fornecido pela GEV;

4. A nova posição poderia se manter armada até que a razão atingisse o quantil inferior indicado pela GEV, o que aconteceu no dia 28/9/2007 (período 61), trinta e um dias úteis após a inversão. O gráfico da Figura 7 mostra a rentabilidade acumulada a partir da inversão das posições armadas.

Ao se analisar a estratégia como um todo, obteríamos uma rentabilidade conjunta de 12,19\% para um período de 36 dias úteis.

PETR4.SA (LONG) E VALE5.SA (SHORT)

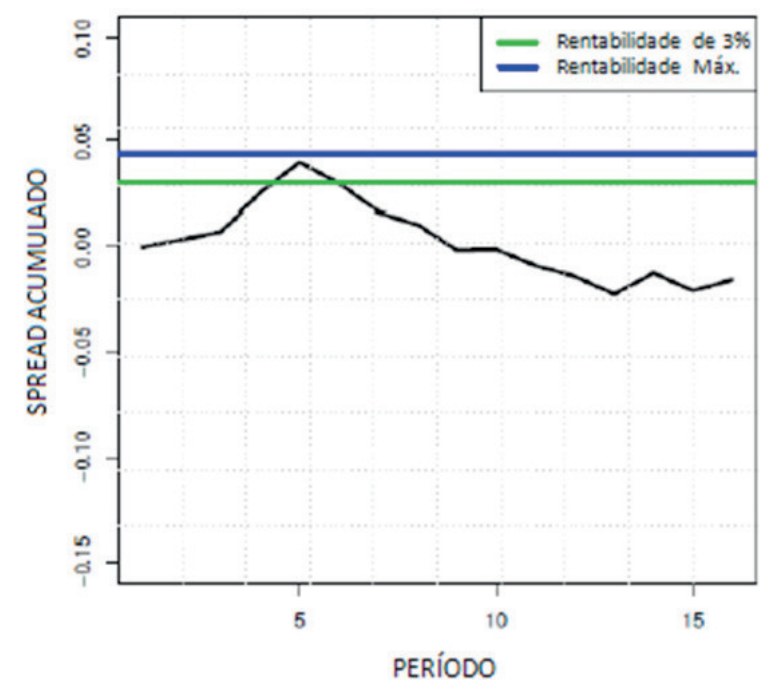

Figura 6. Rentabilidade acumulada das posições das a partir do quantil da normal
PETR4.SA (SHORT) E VALE5.SA (LONG)

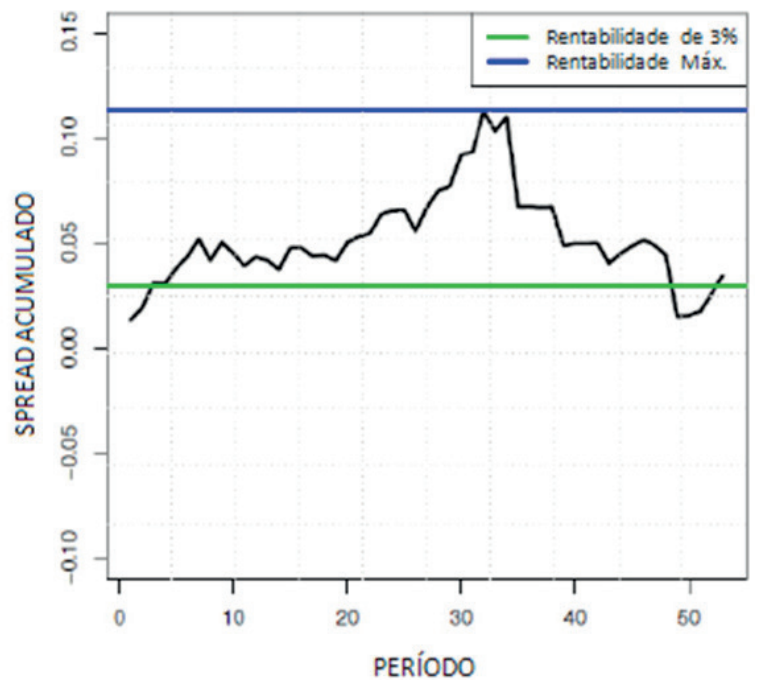

Figura 7. Rentabilidade acumulada das posições a partir da inversão da posições

A mesma análise foi desenvolvida para o par VIVO4 e TCSL4, cujos dados coletados formam uma amostra de 396 observações dos valores diários dos preços desses ativos para o período de 1\%/6/2006 a 15/12/2007. Neste caso, tanto na modelagem dos máximos quanto na dos mínimos os blocos de tamanho 5 garantiram maior max-estabilidade para a GEV.

A partir dos parâmetros obtidos, a GEV ajustada para mínimos e máximos fornece 0,9042828 e 1,455642 como quantis $5 \%$ e $95 \%$ para a razão $\mathrm{k}=$ VIVO4/TCSL4, respectivamente. Ao se utilizar uma distribuição normal, obtêm-se para o mesmo nível de significância os valores 0,9285019 e 1,424783, respectivamente. Na Figura 8 são comparados os quantis de probabilidade obtidos com a performance da razão k para os 54 dias subsequentes ao período analisado. Na Figura 8 estão também inclusas as inferências acerca dos verdadeiros quantis de probabilidade, a saber 1,052407 e 1,505114, respectivamente. 


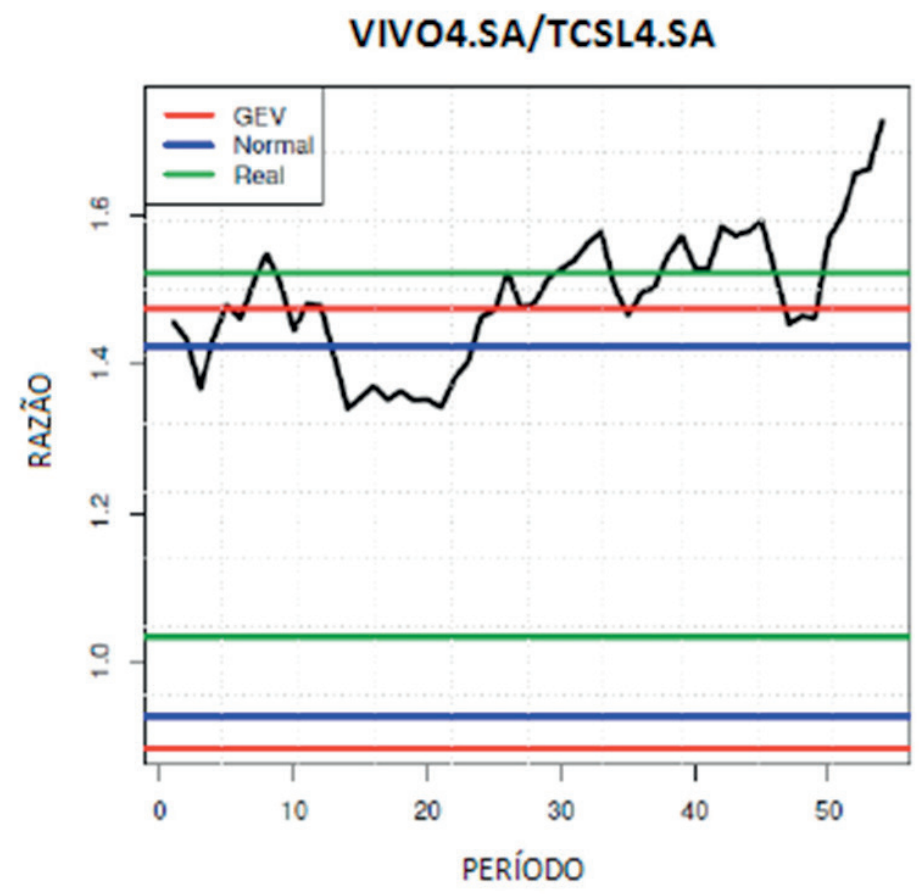

Figura 8. Performance da razão $k=P$ ETR4.SA/VALE5.SA para os 54 dias subsequentes ao período compreendido entre $01 / 06 / 2006$ a 15/12/2007

Caso os players tivessem conhecimento acerca dos quantis estimados pela normal e GEV, a partir do monitoramento diário da evolução da razão entre os ativos a seguinte estratégia de arbitragem poderia ser armada:

1. Ao acompanhar-se a evolução da razão $\mathrm{k}=\mathrm{VIVO} 4 / \mathrm{TCSL} 4$, verifica-se que no período 2 a razão de precificação $\mathrm{k}$ mantém sua tendência de queda e assume valor bastante próximo do quantil $95 \%$ fornecido pela distribuição normal. Embora a razão esteja abaixo dos suportes superiores da GEV, o valor de k se encontra próximo desses suportes, o que reforça a expectativa de queda continuada da razão de precificação. Diante disso, poder-se-ia montar posições comprada em TCSL4 e vendida em VIVO4, fixando-se uma rentabilidade acumulada de $2 \%$ para inversão das posições. A partir desse limite, verifica-se que tal inversão aconteceria ao final do mesmo dia, ou seja, um dia útil após o start da operação (ver Figura 9), a partir da obtenção de uma rentabilidade acumulada de 2,04\%.

2. Ao se alcançar a rentabilidade desejada, assume-se posição comprada em VIVO4 e vendida em TCSL4. Note que a inversão das posições se justifica pela reação natural do mercado à tendência de queda contínua à qual a razão $\mathrm{k}$ foi submetida.

3. Uma vez armadas as novas posições, pode-se determinar o ponto de stop da operação quando $\mathrm{k}$ atinja o real suporte superior da distribuição dos dados ou quando seja obtida uma rentabilicade acumulada de $2 \%$, contada a partir do ponto em que $\mathrm{k}$ rompe o limite superior fornecido pela GEV. Nesse caso, esse momento se mostra presente 5 dias úteis após o início da inversão, quando a razão de precificação atinge o valor real do suporte da distribuição. Para esse período, a operação fornece rentabilidade acumulada de 5,38\% (ver Figura 10), a qual é igual à rentabilidade máxima que poderia ser obtida no período. Esse fato é mais um dos fatores que comprova o melhor ajuste dos quantis extremos a partir da GEV. 
TCSL4.SA (LONG) E VIVO4.SA (SHORT)

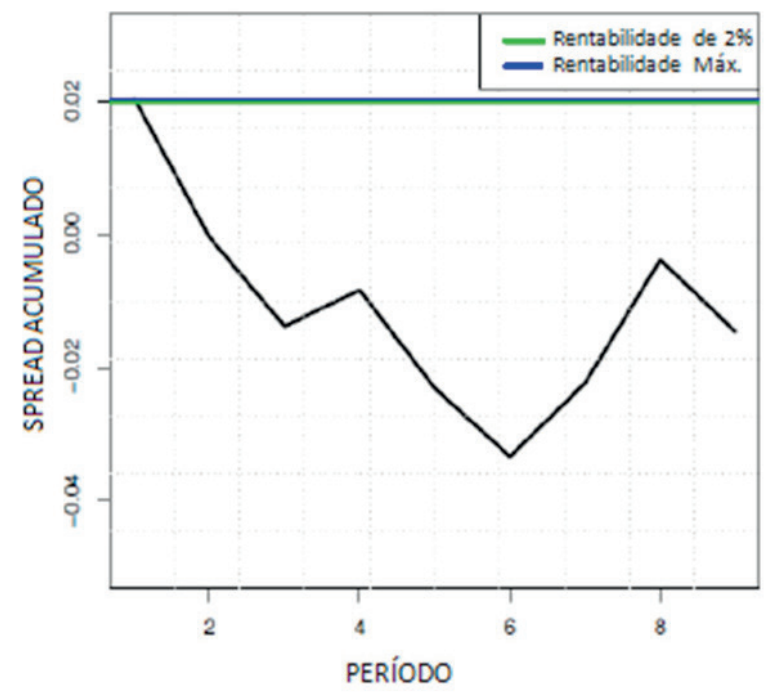

Figura 9. Rentabilidade acumulada das posições a partir do quantil da normal
TCSL4.SA (SHORT) E VIVO4.SA (LONG)

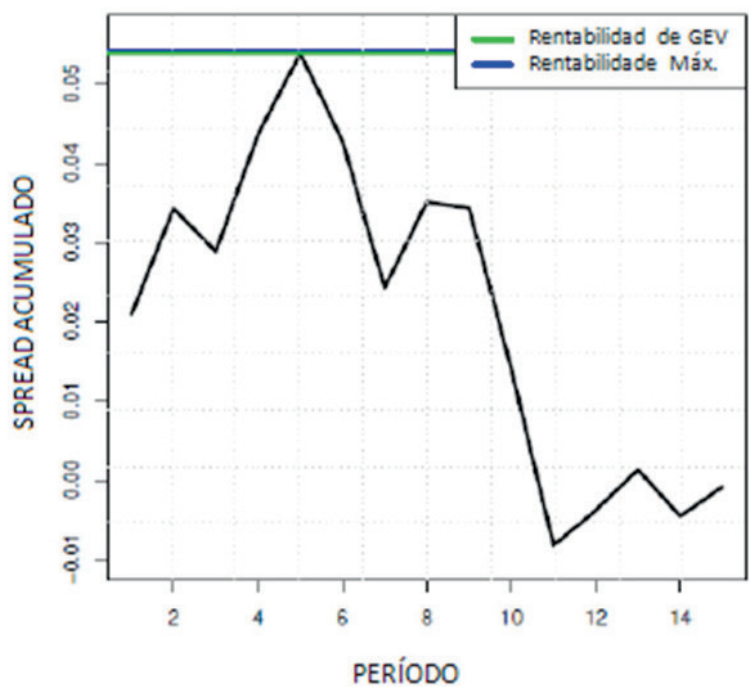

Figura 10. Rentabilidade acumulada a partir da inversão das posições

Ao se analisar a estratégia como um todo, obtém-se uma rentabilidade conjunta de 7,42\% para um período de 6 dias úteis. A estratégia utilizada poderia ainda ser ampliada de modo que fossem invertidas novamente as posições, uma vez que o suporte superior da real distribuição dos dados foi alcançado. Poder-se-ia determinar o ponto de stop da operação caso seja obtida uma rentabilidade acumulada de $2 \%$ contada a partir do ponto em que $\mathrm{k}$ rompe o limite superior fornecido pela GEV. Caso tal inversão fosse utilizada, seriam incorporados à rentabilidade conjunta da operação 3,99\% em 5 dias úteis.

Árbitros mais agressivos poderiam adotar como momento de stop da estratégia acima o ponto em que seja obtida uma rentabilidade acumulada de $2 \%$ contada a partir do momento em que k rompe o limite superior fornecido pela normal (e não pela GEV). Observe que, caso as expectativas desses árbitros se confirmassem, o mercado retornaria à situação inicial em que se encontrava no início de toda a estratégia, uma vez que estaria em tendência de queda e rompendo o quantil superior fornecido pela normal. Note que esse fato se confirma para o período analisado.

\section{Conclusão}

No universo quantitativo da detecção de janelas de arbitragem não se pode considerar que todos os investidores estejam de acordo quanto à distribuição de probabilidade dos preços dos ativos. Isso porque, na maior parte dos casos, a verdadeira distribuição dos preços, bem como dos respectivos retornos, é desconhecida. O que os investidores fazem, na verdade, é aproximar em função das informações que têm disponíveis a distribuição empírica dos dados para distribuições conhecidas e que melhor se adequem ao tipo de análise requerida. Esse fato faz com que não sejamos capazes de assegurar, na prática, a existência de um conjunto de carteiras e de estratégias que sejam eficientes a todos os investidores (o que não implica a não existência teórica da fronteira eficiente e de estratégias maximizadoras de rentabilidade). Por consequência são criadas janelas de arbitragem, dadas as diferentes expectativas que cada investidor faz acerca da carteira ótima para alocação de seus recursos, das tendências de evolução dos preços dos ativos financeiros e das estratégias a serem utilizadas.

A velocidade e a eficiência da interpretação das informações por parte dos investidores também não podem ser consideradas homogêneas, ainda que se considere que as informações estejam instantaneamente disponíveis a todos os investidores. 
A defasagem temporal causada pelo fator processamento e a qualidade de interpretação causada pelo fator eficiência fazem com que não se possa considerar ainda, como o faz as finanças modernas, as hipóteses de racionalidade ilimitada e informação perfeita. Nesse sentido, os preços dos ativos financeiros não refletem em cada momento toda a informação disponível, mas o conjunto de interpretações que cada investidor faz dessas informações.

Nesse sentido, a busca por metodologias de análise que melhor incorporem as reações dos investidores frente às alterações do comportamento do mercado se tornam cada vez mais necessárias. Conforme verificado, por meio dos exemplos anteriores, a modelagem (por meio da TVE) das séries obtidas, a partir dos quocientes dos preços dos pairs trading arbitrados mostrou que a GEV melhor ajustou os quantis de probabilidades extremos. Esse resultado foi obtido ao detectar, com 95\% de confiança, um timing mais oportuno para a reversão da tendência de queda e de aumento da razão de precificação entre os pares de ativos escolhidos.

O par de ativos PETR4 e VALE5 confere grande representatividade no total de negociações do mercado brasileiro. Embora exista um sincronismo aparente entre os preços dos dois ativos considerados, verificou-se a partir desse exemplo que a condição de cointegração não é necessariamente um requisito para que estratégias long-short possam ser construídas, como é o caso da GEV Long-Short Strategy proposta. Tal fato se sustenta, uma vez que a modelagem probabilística da razão de precificação, por meio da GEV, não procura estabelecer relação de dependência entre os processos geradores de cada série de preços dos ativos, mas se propõe a verificar probabilisticamente. Assim, a partir da existência de cada processo gerador, momentos em que a razão de precificação ocupa quantis extremos e se mostra fora dos padrões de normalidade. Essa interpretação confere à metodologia de análise um avanço em termos de oportunidades de arbitragem com relação à metodologia que utiliza cointegração, uma vez que o fato de séries de preços de ativos não serem cointegradas não exclui a possibilidade da existência de janelas de arbitragem em curto prazo.

Além das rentabilidades positivas obtidas para os pares de ativos considerados, os resultados sugerem que a modelagem a partir da GEV é capaz de fornecer informações acerca de janelas de arbitragem em curto prazo, mesmo para pares de ativos que não possuam interdependência de longo prazo. Os resultados sugerem ainda que o melhor ajuste dos quantis extremos por meio da GEV tem capacidade de fornecer sustentação probabilística mais refinada para que o retorno à média justifique a possibilidade de arbitragem long-short. É importante observar, entretanto, que, embora a modelagem dos dados por meio da distribuição normal tenha superestimado os quantis inferiores e subestimado os quantis superiores, tais quantis sinalizaram tendências secundárias de crescimento para os pares de ativos considerados.

A utilização dos quantis teóricos da GEV pode fornecer informações para que seja feito um monitoramento ex-ante da difusão gradual das informações privadas e dos respectivos ajustes relativos dos preços. Tal monitoramento torna-se importante na medida em que possibilita que os players revejam suas expectativas e percebam com pequena defasagem temporal o timing oportuno em que devem operar como traders. As altas performances obtidas por meio do monitoramento das duas ações de maior peso do mercado brasileiro e de duas ações importantes no crescente setor de comunicações confirmam a importância da nova estratégia quantitativa long-short proposta como metodologia de análise dentro do mercado de arbitragem. 


\section{Referências}

Abarbanell, J. S. \& Bushee, B. J. (1998). Abnormal return to a fundamental analysis strategy. The Accounting Review. 73 (1), pp. 19-45.

Abarbanell, J. S. \& Bushee, B. J. (1997). Fundamental analysis, future earnings, and stock prices. Journal of Accounting Research, 35(1), pp. 1-24.

Alexander, C. (1999). Optimal Hedging Using Cointegration. Philosophical Transactions of the Royal Society, London, Series A, 1(357), pp. 2039-2058. Recuperado em 11/04/2014 de http://www.icmacentre.ac.uk/pdf/cointegration.pdf.

Alexander, C. \& Dimitriu, A. (2005a). Indexing and statistical arbitrage: tracking error or cointegration? Philosophical Transactions of the Royal Society, Journal of Portfolio Management, 31(1), pp. 5063-5078.

Alexander, C. \& Dimitriu, A. (2005b). Indexing, cointegration and equity market regimes. International Journal of Finance and Economics, 10(3), pp. 213-231.

Ali, A. \& Hwang, L. (2000). Country-specific factors related to financial reporting and the value relevance of accounting data. Journal of Accounting Research, 31(1), pp. 1-21.

Anjivel, S.I., Boudreu, B.E., Perskin, M.W. \& Urias M.S. (2000). Why hedge funds make sense? Quantitative Strategies Research Memorandum, Morgan Stanley.

Ball, R. \& Brown, P. (1968). An Empirical evaluation of accounting income numbers. Journal of Accounting Research, 6(2), pp. 159-178.

Baruch, L. \& Thiagarajan, S. R. (1993). Fundamental information analysis. Journal of Accounting Research, 31(2), pp. 190-215.

Bird, R., Gerlach, R. \& Hall, A. D. (2001). The prediction of earnings movements using accounting data: an update and extension of $\mathrm{Ou}$ and Penman. Journal of Asset Management, 2(2), pp. 180-195. doi:10.1057/palgrave.jam.2240043

Bortkiewicz, L. V. (1922). Variationsbreite und mittlerer Fehler. Sitzungsber, Berli. Math. Gess, 3(21), pp. 3-11.

Branco, G.C. \& Franco, D. (2004). Risco e retorno nos hedge funds brasileiros. $4^{\circ}$ Encontro da Sociedade Brasileira de Finanças - SBFIN. Rio de Janeiro.

Caldeira, J.F. \& Portugal, M.S. (2010). Estratégia Long-Short, Neutra ao Mercado, e Index Tracking Baseadas em Portfólios Cointegrados. Revista Brasileira de Finanças, 8(4), pp. 469-504.

Dunis, C.L. \& Ho, R. (2005). Cointegration portfolios of european equities for index tracking and market neutral strategies. Journal of Asset Management, 1(1), pp. 33-52.

Do, B.F. \& Hamza, K. (2006). A new approach to modeling and estimation for pairs trading. Working Paper Series. Monash University. Recuperado em 11/04/2014 de https://statarb.googlecode.com/ files/0PairsTrading_BinhDo.pdf.

Embrechts, P., Kluppelberg, C. \& Mikosch, T. (1997). Modelling extremal events for insurance and finance. Springer-Verlag: Berlin.

Fama, E. F. \& French, K. R. (1992). The cross section of expected stock returns. The Journal of Finance, 47(2), pp. 427-465.

Fama, E. F. \& French, K. R. (1995). Size and book-to-market factors in earnings and returns. The Journal of Finance, 50(1), pp. 131-155.

Fama, E. F. \& French, K. R. (1996). Multifactor explanations of asset pricing anomalies. Journal of Finance, 51(1), pp. 55-84. 
Fisher, R. A. \& Tippett, L. H. C. (1928). Limiting Forms of the Frequency Distribution of the Largest or Smallest Member of a Sample. Proccedings of the Cambridge Philosophical Society, 1(24), pp. 180-190.

Fothergill, M. \& Coke, C. (2000). Funds of Hedge Funds. An Introduction to Multi-manager Funds. Deutsche Bank.

Gatev, E., Goetzmann, W. \& Rouwenhorst, K. (2006). Pairs trading: Performance of a relative-value arbitrage rule. Review of Financial Studies 3(19), pp. 797-827.

Gumbel, E. J. (1954). Statistics Theory of Extreme Values and Some Pratical Applications. Nat. Bureau of Standards Applications Mathmatics Series, 2(33), pp. 1-51.

Gumbel, E. J. (1958). Statistics of Extremes. Columbia University Press, New York Journal of Business, 1(63), pp. 383-408.

Herlemont, D. (2000). Pairs Trading, Convergence Trading, Cointegration. YATS Working Papers. YATS Finances Technologies.

Jenkinson, A. F. (1955). The frequency distribution of the annual maximum (or minimum) values of meteorological elements. Quart. J. Roy. Meteo. Soc. 1(81), pp. 158-171.

Liang, B. (2003). Hedge fund returns: Auditing and accuracy. The Journal of Portfolio Management 29(3), pp. 111-122.

Lin, Y., McRae, M. \& Gulati, C. (2006). Loss Protection in Pairs Trading through Minimum Profit Bounds: A Cointegration Approach. Journal of Applied Mathematics and Decision Sciences, 6(16), pp. 114-130.

Lopes, A. B. \& Galdi, F. C. (2007). Financial statement analysis generate abnormal returns under adverse conditions? In: Annual Meeting of the American Accounting Association. Chicago, Illinois.

Loomis C. J. (1966). The Jones Nobody Keeps Up With. Fortune Magazine, pp. 237-248.

Nath, P. (2006). High Frequency Pairs Trading with U.S Treasury Securities: Risks and Rewards for Hedge Funds. Working Paper Series. London Business School.

Nicholas, J. G. (2000). Market-Neutral Investing: long/short hedge funds strategies. Bloomberg Professional Lybrary, New York, ed. 1.

Piotroski, J. D. (2000). Value investing: the use of historical financial statement information to separate winners from losers. Journal of Accounting Research, 38, pp. 1-41.

Sanfins, M. A. S. (2009). Copulas para distribuições generalizadas de valores extremos multidimensionais. Rio de Janeiro: UFRJ/IM.

Von Mises, R. (1936). La distribution de la plus grande de n valeurs. Reprinted in Selected Papers Volumen II, American Mathematical Society, Providence, R.I., 1954, pp. 271-294. 